Research Article

\title{
The Protective Effect of Aspirin against Myocardial Hypertrophy in Rats
}

\author{
Xiaolong Wu $\mathbb{D},{ }^{1,2}$ Minghui Wei $\mathbb{D}^{1},{ }^{1}$ Haifeng Zhang $\left(\mathbb{D},{ }^{3}\right.$ Xiaomei Fan $\mathbb{D}^{1},{ }^{1}$ Xiaochen $M a\left(\mathbb{D},{ }^{1}\right.$ \\ Jiaming Liu $\mathbb{D}^{1}$, and Mingming Xue $\mathbb{D}^{1}$
}

${ }^{1}$ Department of Physiology, Inner Mongolia Medical University, Hohhot, Inner Mongolia 010110, China

${ }^{2}$ Department of Laboratory, Baotou Central Hospital, Baotou city, Inner Mongolia 014040, China

${ }^{3}$ School of Basic Sciences, Inner Mongolia Medical University, Hohhot, Inner Mongolia 010110, China

Correspondence should be addressed to Mingming Xue; happybird-nmg@163.com

Xiaolong Wu and Minghui Wei contributed equally to this work.

Received 20 August 2020; Revised 1 February 2021; Accepted 20 March 2021; Published 21 April 2021

Academic Editor: Inna Gladysheva

Copyright ( 2021 Xiaolong Wu et al. This is an open access article distributed under the Creative Commons Attribution License, which permits unrestricted use, distribution, and reproduction in any medium, provided the original work is properly cited.

\begin{abstract}
The protective effect of aspirin against myocardial hypertrophy $(\mathrm{MH})$ was studied. Model rats of pressure overload $\mathrm{MH}$ were prepared by abdominal aortic coarctation. Rats were randomly divided into the sham group $(n=9)$, MH model group ( $n=9)$, and $\mathrm{MH}+$ aspirin group $(n=9)$, which was, respectively, divided into the 4-week group and 8-week group according to the time of intragastric administration. Arterial blood pressure and left ventricular mass index (LVMI) were measured. Changes in myocardial tissue structure were observed by HE staining, Masson staining, and reticular fiber staining. Cardiomyocyte apoptosis was detected by TUNEL assay. The levels of TNF- $\alpha$, IL-10, TXA2, and PGI2 in myocardium and plasma were detected by ELISA. The arterial blood pressure in the MH model group was significantly higher than that in the 4- and 8-week sham groups, but that in the MH+aspirin group was significantly lower than that in the MH model group. At 4 and 8 weeks, the LVWI in the $\mathrm{MH}$ model group was significantly higher than that in the sham group, but it was significantly reduced after aspirin treatment. The myocardial cell hypertrophy was obvious, collagen fibers were proliferated, and reticular fibers were reduced in the 4- and 8-week MH model groups. Compared with the MH model groups, myocardial cells in the $\mathrm{MH}+$ aspirin groups were significantly reduced, the collagen fiber content was significantly reduced, and the reticular fiber content was increased. The apoptotic cardiomyocytes in the 4- and 8-week $\mathrm{MH}$ model groups were obviously increased. The apoptosis of myocardial cells in the MH+aspirin groups was obviously decreased. The TNF- $\alpha$ levels in the myocardial tissue of the 4- and 8-week MH model groups were significantly increased, while those of the MH+aspirin groups were significantly decreased. There was no significant change in the IL-10 level or PGI2 level at 4 weeks. At 8 weeks, the PGI2 level was significantly decreased in the MH model group while significantly increased in the MH+aspirin group. The TXA2 levels were significantly increased in the 4- and 8-week MH model groups and those in the 4- and 8-week MH+aspirin groups were significantly lower. Aspirin has an anti-inflammatory effect, can effectively reduce the expression of inflammatory factors, inhibit myocardial apoptosis, and has a certain protective effect against $\mathrm{MH}$.
\end{abstract}

\section{Introduction}

Myocardial hypertrophy ( $\mathrm{MH})$, an adaptive response to myocardial overload, is one of the independent risk factors for various cardiovascular diseases [1]. It is characterized by the proliferation of myocardial connective tissue and the increase of cell volume. The persistent $\mathrm{MH}$ is often an early manifestation of heart failure [2]. The pathogenesis of $\mathrm{MH}$ is unclear. Studies have shown that it may be related to inflammation, neurohumoral factors, and endocrine factors $[3,4]$.

Studies have shown that a large number of inflammatory factors participate in the formation of $\mathrm{MH}$ (including ventricular remodeling, fibrosis, and apoptosis) [5], including tumor necrosis factor (TNF-a) and interleukin 6 (IL-6) and 
other anti-inflammatory factors such as interleukin 10 (IL-10) [6]. It is currently believed that TNF- $\alpha$ has effects on many aspects of MH. TNF- $\alpha$ can induce cardiomyocytes to undergo apoptosis, cause cardiomyocytes to hypertrophy, and can bidirectionally regulate the production and degradation of collagen fibers. IL-10 is a major anti-inflammatory factor that inhibits the synthesis and biological effects of many inflammatory factors [7]. The regulation between inflammatory factors is very complex. Proinflammatory and anti-inflammatory factors can both promote each other and antagonize each other. They maintain a balance to maintain the body's homeostasis [8].

Cyclooxygenase (COX) has two isoforms COX-1 and COX-2. COX-1 plays a role in homeostasis and is constitutively expressed. However, COX-2 expression can be induced by inflammation [9]. Thus, COX-2 is an important proinflammatory cell factor and is associated with proinflammatory factors such as TNF- $\alpha$ and IL-6 and anti-inflammatory factors such as IL-10 [9]. Aspirin is a nonselective COX inhibitor that blocks COX and reduces the production of Prostaglandin (PG) and ThromboxaneA2 (TXA2) [10, 11]. Aspirin inhibits the metabolism of arachidonic acid by inhibiting the activity of COX and finally inhibits the synthesis of TXA2 and PG to achieve anti-inflammatory effects $[12,13]$. Aspirin is used for the prevention of many types of diseases, including cardiovascular disease [14].

Apoptosis is involved in many cardiovascular diseases such as hypertension, cardiac hypertrophy, and atherosclerosis [15]. During the development of $\mathrm{MH}$, cardiomyocyte apoptosis plays an important role.

In this study, we aim to investigate the protective effect of aspirin against $\mathrm{MH}$. The changes of inflammatory factors during the development of $\mathrm{MH}$ were analyzed. The effects of aspirin on the left ventricular structure of $\mathrm{MH}$ rats and the relationship between aspirin and inflammatory factors TNF- $\alpha$, IL-10, PGI2, and TXA2 were investigated.

\section{Materials and Methods}

2.1. Animals. Wister male rats weighing about $230 \mathrm{~g}(n=54$; 10 -week-old) were obtained from the Animal Experimental Center of Inner Mongolia University. All animal experiments were conducted according to the ethical guidelines of Inner Mongolia University (approval number: SCXK(Mongolia)2016-0001).

2.2. Model Establishment and Grouping. $\mathrm{MH}$ was established by abdominal aortic coarctation [16]. Briefly, rats were anesthetized with $2 \%$ pentobarbital sodium (i.p.; $40 \mathrm{mg} / \mathrm{kg}$ ). A midline incision was made 2 to $3 \mathrm{~cm}$ below the xiphoid of rats. After exposing the abdominal cavity, the peritoneum, and the left kidney, the abdominal aorta was isolated. The ligation was performed at 0.5 to $1.5 \mathrm{~cm}$ of the abdominal aorta where an obvious pulsation could be felt. The pentobarbital sodium was also used for postoperative analgesia. The surgery success rate was over $80 \%$ with $1-2$ deaths in each group. After model establishment, the rats were randomly divided into the MH model group $(n=18)$ and $\mathrm{MH}+$ aspirin group $(n=18)$. In the sham group $(n=18)$, the same proce- dures were performed but without ligation. Rats in the $\mathrm{MH}$ model and the sham group were intragastrically administered with normal saline on the next day after operation. Rats in the $\mathrm{MH}+$ aspirin group were intragastrically administered with $10 \mathrm{mg} / \mathrm{mL}$ aspirin solution $(200 \mathrm{mg} / \mathrm{kg} /$ day $)$. Aspirin was administered chronically for 4 or 8 weeks. The above three groups were randomly divided into the 4-week group and 8-week group according to the time of intragastric administration.

2.3. Noninvasive Arterial Blood Pressure Measurement. The systolic blood pressure (SBP) and diastolic blood pressure (DBP) of each group were measured by automatic noninvasive blood pressure measuring instrument BP-300A (CDTME, Chengdu, China) at 4 and 8 weeks after operation. The arterial blood pressure values were measured 3 times and averaged.

2.4. Collection of Cardiac Blood and Myocardial Tissue Specimens. At the end of the experiment, the rats were anesthetized with $2 \%$ sodium pentobarbital. The cardiac blood was collected from the heart, and the serum was isolated. After that, the rats were sacrificed by decapitation. Then, the heart was dissected, blotted by the filter paper, and weighed. The left ventricle was weighed, and the left ventricular mass index (LVMI) was calculated. The myocardial tissue was taken along the long axis of the left ventricle. Some of the myocardial tissue was fixed with $10 \%$ neutral formalin and made into paraffin sections. Some of the myocardial tissue was stored in liquid nitrogen, which was used in ELISA to detect the content of inflammatory factors in myocardial tissue.

2.5. Morphological Changes of Left Ventricular Structure. HE staining, Masson staining, and modified Gomori reticular fiber staining were used to observe left ventricular morphological structure and fiber changes. The protocols were performed according to routine procedure. Briefly, for $\mathrm{HE}$ staining, the sections were dewaxed, stained with hematoxylin-eosin, dehydrated and transparentized, and mounted. For Masson's trichrome staining, the sections were dewaxed, stained with hematoxylin followed by Masson staining, dehydrated and transparentized, and mounted. For the modified Gomori reticular fiber staining, the sections were dewaxed, stained with Gomori ammonia silver, dehydrated and transparentized, and mounted. Finally, the morphological changes were observed under light microscope.

To calculate the average cardiomyocytes diameter, 10 fields of each specimen were randomly selected under a 400x microscope, and 10 cells were selected from each field. The area of each cell was measured with Image-Pro Plus, and the average diameter was calculated after averaging.

To calculate the collagen volume fraction (CVF), the Leica Application Suite-LAS system was used to process the image under 200x magnification. One slice was selected for each specimen, and 6 regions without coronary vessels and 6 regions with coronary vessels were selected for each slice. CVF was calculated as the average percentage of collagen fibers. The CVF of the coronary arterial visual field was 
TABLE 1: Measurement of arterial blood pressure in each group (mean $\pm \mathrm{SD}, n=9(\mathrm{mmHg})$ ).

\begin{tabular}{|c|c|c|c|c|}
\hline \multirow{2}{*}{ Groups } & \multicolumn{2}{|c|}{ SBP } & \multicolumn{2}{|c|}{ DBP } \\
\hline & 4-week & 8-week & 4-week & 8-week \\
\hline Sham & $108.6 \pm 5.45$ & $110.4 \pm 8.67$ & $90.7 \pm 4.17$ & $85.6 \pm 7.97$ \\
\hline MH model & $122.2 \pm 13.07^{*}$ & $128.9 \pm 13.27^{*}$ & $99.1 \pm 6.28^{*}$ & $108.1 \pm 9.37^{*}$ \\
\hline $\mathrm{MH}+$ aspirin & $110.2 \pm 6.15^{\triangle}$ & $108.9 \pm 6.97^{\triangle}$ & $91.4 \pm 6.51^{\triangle}$ & $90.9 \pm 5.32^{\triangle}$ \\
\hline
\end{tabular}

Note: ${ }^{*} P<0.05$ compared with the sham group at the same time point. ${ }^{\triangle} P<0.05$ compared with the MH model group at the same time point.

TABLE 2: Measurement of left ventricular weight, body weight, and LVMI in each group (mean \pm SD, $n=9$ ).

\begin{tabular}{|c|c|c|c|c|c|c|}
\hline \multirow[t]{2}{*}{ Groups } & \multicolumn{2}{|c|}{ Left ventricular weight (g) } & \multicolumn{2}{|c|}{ Body weight (g) } & \multicolumn{2}{|c|}{$\begin{array}{l}\text { Left ventricular weight/body } \\
\text { weight } \times 10^{3} \text { (LVMI) }\end{array}$} \\
\hline & 4-week & 8-week & 4-week & 8-week & 4-week & 8-week \\
\hline Sham & $0.62 \pm 0.08$ & $0.71 \pm 0.05$ & $293.3 \pm 23.50$ & $344.8 \pm 29.56$ & $1.9 \pm 0.28$ & $2.2 \pm 0.23$ \\
\hline MH model & $0.74 \pm 0.10^{*}$ & $0.83 \pm 0.11^{*}$ & $275.2 \pm 16.06$ & $296.2 \pm 17.75^{*}$ & $2.4 \pm 0.21^{*}$ & $2.9 \pm 0.27^{*}$ \\
\hline $\mathrm{MH}+$ aspirin & $0.65 \pm 0.17^{\triangle}$ & $0.72 \pm 0.17^{\triangle}$ & $284.7 \pm 11.17$ & $322.8 \pm 11.52$ & $2.0 \pm 0.13^{\triangle}$ & $2.4 \pm 0.11^{\triangle}$ \\
\hline
\end{tabular}

Note: LVMI: left ventricular mass index; ${ }^{*} P<0.05$ compared with the sham group at the same time point. ${ }^{\triangle} P<0.05$ compared with the MH model group at the same time point.

defined as the percentage of total left ventricular myocardial collagen volume fraction (CVF-T). The visual field collagen volume fraction percentage was abbreviated as (CVF-NV).

One section was selected for each specimen, and six fields of view were selected for each section. The volume percentage of the reticular fibers in each field of vision was calculated and defined as volume percentage (RVF-T).

2.6. TUNEL. Cardiomyocyte apoptosis was detected by TUNEL assay according to the manufacturer's instructions (Promega, Madison, Wisconsin, USA). The nuclei were stained with DAPI. The green fluorescence, which indicates the apoptosis, was observed at $520 \pm 20 \mathrm{~nm}$. The blue fluorescence, which indicates the nuclei, was observed at $460 \mathrm{~nm}$. The apoptosis rate was calculated as number of apoptotic cells (TUNEL positive)/total number of cells (DAPI positive) $\times 100 \%$.

2.7. ELISA. The cardiac blood was collected from the heart, and the serum was isolated. The myocardial tissue was homogenized, and the supernatant was collected after centrifugation at $5000 \mathrm{r} / \mathrm{min}$ for $15 \mathrm{~min}$. The levels of IL-10, PGI $\mathrm{TXA}_{2}$, and TNF- $\alpha$ in the serum and myocardial tissue supernatant were determined with a Rat ELISA test kit according to the manufacturer's instructions (Biowinner, Beijing, China). HRP-labeled goat anti-rabbit IgG $(1: 5)$ were used as the secondary antibody. The absorbance value at $450 \mathrm{~nm}$ wavelength was read with a microplate reader (Multiskan MK-3, Finland).

2.8. Statistical Analysis. The data was analyzed by SPSS 13.0 (IBM, Armonk, NY, USA). Data was described as mean \pm standard deviation. The $t$-test and SNK- $q$ test were used to compare the differences between different groups at the same time point. $P$ value $<0.05$ was considered statistically significant.

\section{Results}

3.1. MH Model Is Established in Rats. To test if the rat model is successfully established, blood pressure was measured at different time points. Compared with the sham group, the blood pressure (SBP and DBP) (Table 1) was increased, and the difference was statistically significant $(P<0.05)$. LVMI was also calculated. As shown in Table 2, LVMI in the MH model group was significantly increased than that in sham group $(P<0.05)$. However, there was a significant decrease in LVMI of the $\mathrm{MH}+$ aspirin group than that of the $\mathrm{MH}$ model group $(P<0.05)$. These showed that the model rats with $\mathrm{MH}$ were successfully established.

3.2. Changes of Ventricular Structure in Model Rats with MH. After HE staining, the myocardial cells in the 4-week MH model group were disordered, hypertrophied, and irregular in morphology (Figure 1). The MH was more obvious, and there were inflammatory cells in the 8-week MH model group (Figure 1). Compared with the $\mathrm{MH}$ model group, the average cardiomyocyte diameter in the 4-week and 8-week $\mathrm{MH}+$ aspirin groups was significantly reduced (Table 3 ).

After Masson staining, the collagen fibers showed focal hyperplasia (mainly perivascular hyperplasia) in the 4-week MH model group (Figure 2). The myocardial injury was more serious, and the collagen fibrosis was more obvious in the 8-week MH model group (Figure 2). The collagen fibers in the 4 - and 8 -week $\mathrm{MH}+$ aspirin groups were more proliferative than the sham group, but not as obvious as the $\mathrm{MH}$ model group (Figure 2). CVF-T and CVF-NV significantly increased in the 4-week and 8-week MH model groups compared with the sham group (Table 4), and the CVF-NV of the 4 -week and 8-week $\mathrm{MH}+$ aspirin groups were significantly lower than the MH model group (Table 4). CVF-T of the $\mathrm{MH}+$ aspirin group decreased significantly compared with the MH model group (Table 4). 

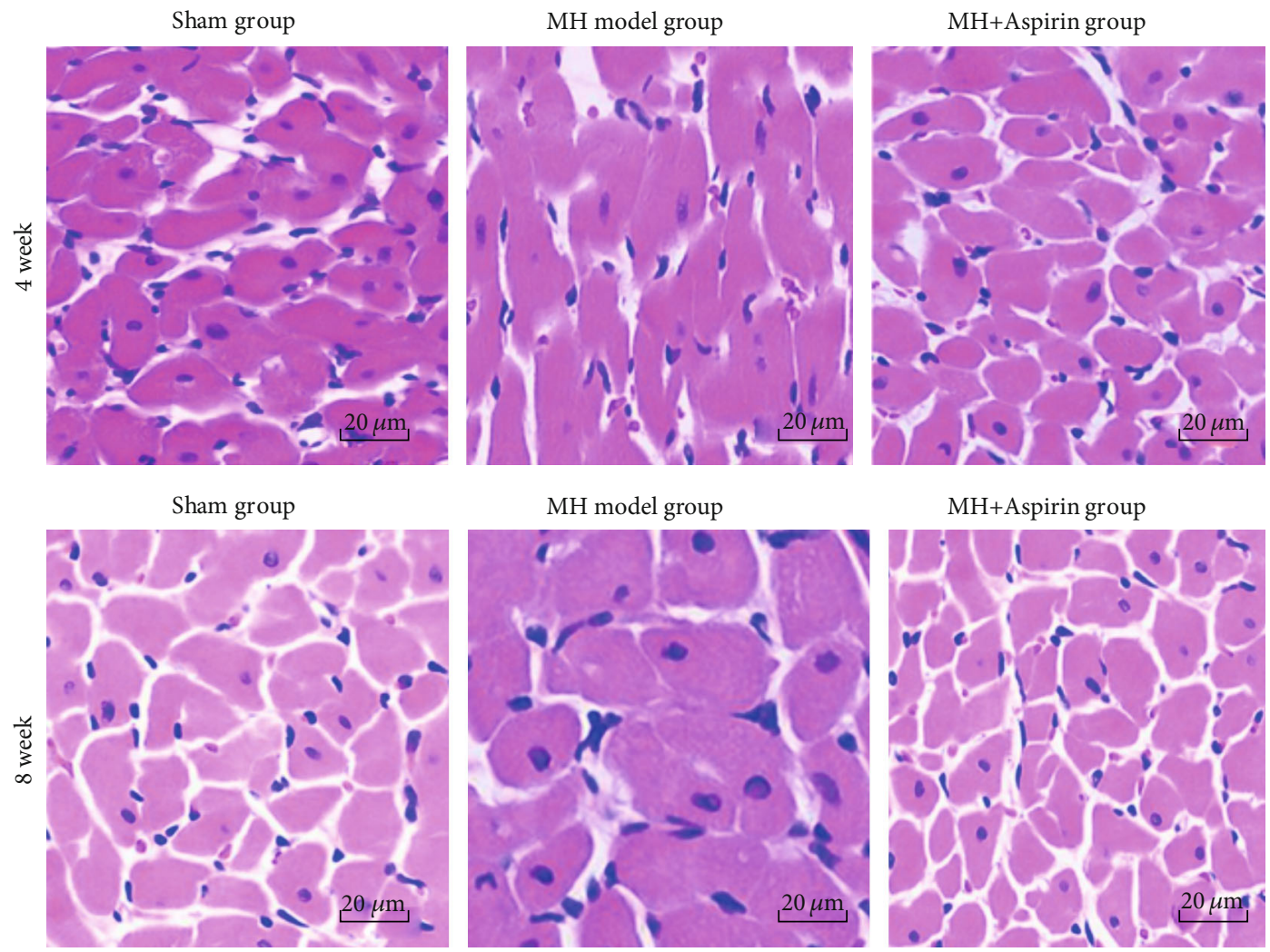

FIGURE 1: Pathological morphology of myocardial tissue in each group $(n=9)$. Myocardial tissues were collected from the sham group, MH model group, and $\mathrm{MH}+$ aspirin group at 4 weeks and 8 weeks after model establishment. HE staining was performed to observe the pathological changes. Representative images were shown. Magnification: 400x.

TABLE 3: Measurement of the average diameter of the myocardial cell in each group (mean $\pm \mathrm{SD}, n=9(\mu \mathrm{m}))$.

\begin{tabular}{lcc}
\hline \multirow{2}{*}{ Groups } & \multicolumn{2}{c}{$\begin{array}{c}\text { Average diameter of myocardial cell } \\
\text { 4-week }\end{array}$} \\
\hline Sham & $9.02 \pm 0.25$ & $9.51 \pm 0.33$ \\
MH model & $9.89 \pm 0.46^{*}$ & $10.18 \pm 0.53^{*}$ \\
MH+aspirin & $9.14 \pm 0.23^{\triangle}$ & $9.65 \pm 0.27^{\triangle}$ \\
\hline
\end{tabular}

Note: ${ }^{*} P<0.05$ compared with the sham group at the same time point. ${ }^{\triangle} P<0.05$ compared with the MH model group at the same time point.

Gomori reticular fiber staining showed that the reticular fibers in the 4- and 8-week $\mathrm{MH}$ model groups were obviously reduced (Figure 3). The 4 -week and 8-week $\mathrm{MH}+$ aspirin group had obviously increased reticular fibers compared with the MH model group (Figure 3). The RVF-T in the 4-week and 8-week MH model groups were significantly less than the sham group (Table 5), and the RVF-T in the 4-week and 8-week $\mathrm{MH}+$ aspirin groups increased significantly compared with the $\mathrm{MH}$ model group (Table 5). These results demonstrate that there is cardiac hypertrophy, collagen fibrosis, and reticular fiber reduction in the early stage of $\mathrm{MH}$.

3.3. Cardiomyocyte Apoptosis in Rats. TUNEL was performed to analyze apoptosis. As shown in Figure 4 and Table 6, compared with the sham group, the apoptotic cardiomyocytes in the 4- and 8-week $\mathrm{MH}$ model groups were obviously increased. Compared with the $\mathrm{MH}$ model group, the apoptosis of myocardial cells in the $\mathrm{MH}+$ aspirin groups was obviously decreased.

3.4. Effect of Aspirin on Arterial Blood Pressure in Model Rats with $M H$. The effect of aspirin on arterial blood pressure in rats with $\mathrm{MH}$ was analyzed. The SBP and DBP were measured at 4 weeks and 8 weeks. Compared with the sham group at the same time point, the SBP and DBP in the $\mathrm{MH}$ model group were increased, and the differences were statistically significant (Table 1). Compared with the $\mathrm{MH}$ model group, the $\mathrm{SBP}$ and $\mathrm{DBP}$ in the $\mathrm{MH}+$ aspirin group were decreased, and the difference was statistically significant (Table 1). These results indicate arterial blood pressure is significantly increased in rats with $\mathrm{MH}$, and this increase was reduced by aspirin.

3.5. Effect of Aspirin on Inflammatory Factors in Model Rats with $M H$. Inflammatory factors were detected by ELISA. The TNF- $\alpha$ level in the serum had no significant difference between the 4 and 8 weeks of the rats in each group (Table 7). The TNF- $\alpha$ levels in the myocardial tissue of the 4- and 8-week $\mathrm{MH}$ model groups were significantly higher than those in the sham groups $(P<0.05)$. The TNF- $\alpha$ levels in myocardial tissue of the 4- and 8-week $\mathrm{MH}+$ aspirin groups were significantly lower than those in the $\mathrm{MH}$ model groups $(P<0.05)$. There was no significant change in the 

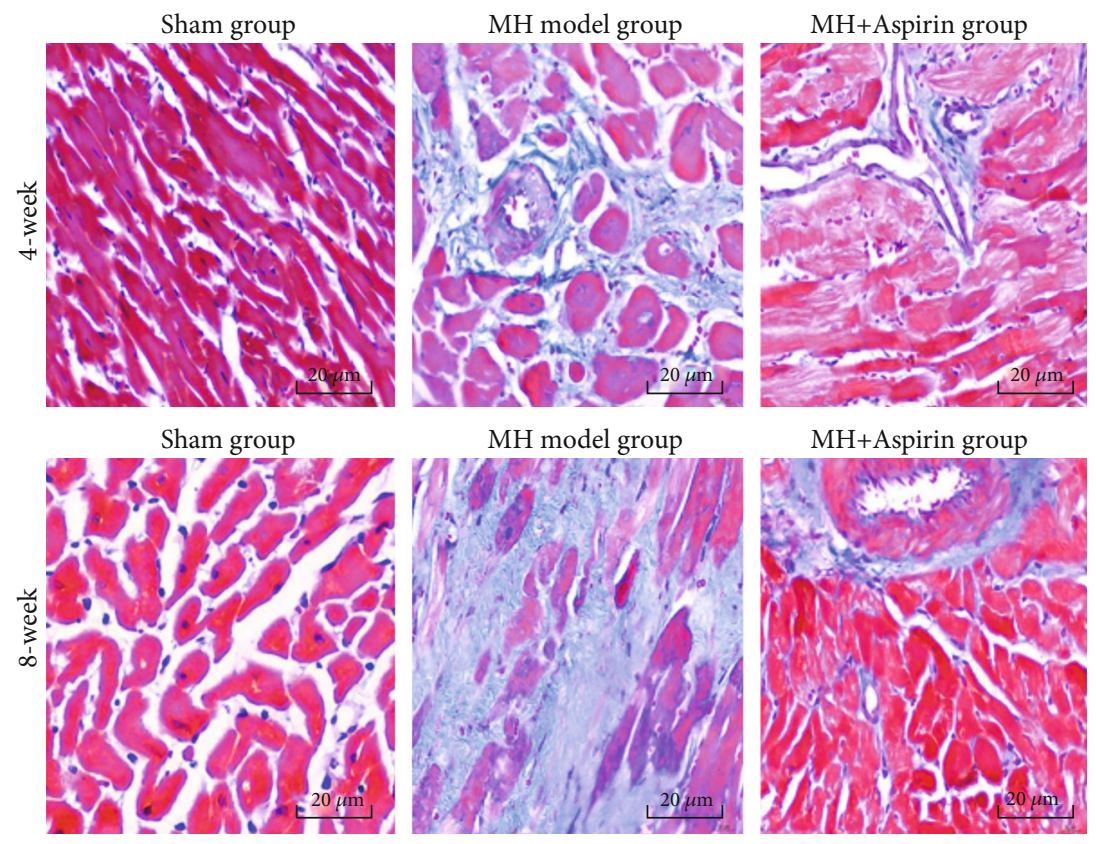

Figure 2: Collagen fiber analysis of myocardial tissue in each group $(n=9)$. Myocardial tissues were collected from the sham group, MH model group, and $\mathrm{MH}+$ aspirin group at 4 weeks and 8 weeks after model establishment. Masson staining was performed to observe the collagen fibers. Representative images were shown. Magnification: 400x.

TABLE 4: Measurement of CVF-NV (\%) and CVF-T (\%) in each group (mean $\pm \mathrm{SD}, n=9$ ).

\begin{tabular}{|c|c|c|c|c|}
\hline \multirow{2}{*}{ Groups } & \multicolumn{2}{|c|}{ CVF-NV (\%) } & \multicolumn{2}{|c|}{ CVF-T (\%) } \\
\hline & 4-week & 8-week & 4-week & 8 -week \\
\hline Sham & $3.35 \pm 0.83$ & $3.43 \pm 0.86$ & $7.95 \pm 1.16$ & $9.76 \pm 1.75$ \\
\hline MH model & $7.16 \pm 2.06^{*}$ & $12.85 \pm 2.03^{*}$ & $27.31 \pm 4.02^{*}$ & $32.05 \pm 6.86^{*}$ \\
\hline $\mathrm{MH}+$ aspirin & $4.64 \pm 2.17^{\triangle}$ & $4.70 \pm 1.34^{\#}$ & $8.46 \pm 1.12^{\#}$ & $11.55 \pm 1.24^{\triangle}$ \\
\hline
\end{tabular}

Note: CVF: collagen volume fraction. CVF-NV: visual field collagen volume fraction percentage; CVF-T: total left ventricular myocardial collagen volume fraction. ${ }^{*} P<0.01$ compared with the sham group at the same time point. ${ }^{\triangle} P<0.05$ compared with the MH model group at the same time point. ${ }^{\#} P<0.01$ compared with the MH model group at the same time point.

IL-10 level in the plasma and myocardial tissue between the 4 and 8 weeks of the rats in each group (Table 8 ). There was no significant change in the $\mathrm{PGI}_{2}$ level in the plasma and myocardial tissue among three groups at 4 weeks (Table 9). The $\mathrm{PGI}_{2}$ level in the plasma and myocardial tissue was significantly decreased in the $\mathrm{MH}$ model group at 8 weeks as compared with that in the sham group $(P<0.05)$. At week 8 , the $\mathrm{MH}$ + aspirin group had significantly higher level of $\mathrm{PGI}_{2}$ than the $\mathrm{MH}$ model group $(P<0.05)$. The $\mathrm{TXA}_{2}$ levels in the plasma and myocardial tissues of the 4- and 8-week $\mathrm{MH}$ model groups were increased as compared with those in the sham group, and the difference was statistically significant (Table 10) $(P<0.05)$. Compared with the $\mathrm{MH}$ model group, the $\mathrm{TXA}_{2}$ levels in the myocardial tissue and plasma of the $\mathrm{MH}+$ aspirin group were significantly lower $(P<0.05)$. These results suggest that TNF- $\alpha$ and TXA2 levels are significantly increased in rats with $\mathrm{MH}$, while the PGI2 level is significantly decreased. Aspirin can significantly reduce the levels of TNF- $\alpha$ and TXA2.

\section{Discussion}

$\mathrm{MH}$ is one of the independent risk factors in cardiovascular diseases. Inflammatory factors play an important role in the development of $\mathrm{MH}$ and are involved in ventricular remodeling [17]. Changes in blood pressure, LVMI, and pathological morphology are the primary indicators of $\mathrm{MH}$ [18]. In this study, we established an MH model according to previous description [19]. The blood pressure and LVMI were observed at 4 weeks and 8 weeks after model establishment. We found that the blood pressure and LVMI in the $\mathrm{MH}$ model group were significantly higher than those in the sham group, indicating that the $\mathrm{MH}$ model is successfully established.

Aspirin can reduce cardiac interstitial fibrosis by inhibiting Erk1/2-Serpine2 and P-Akt signaling pathways [20]. In humans, there is evidence that aspirin may impair reverse myocardial remodeling in heart failure patients treated with beta blockers [21]. Vascular endothelial injury can promote 

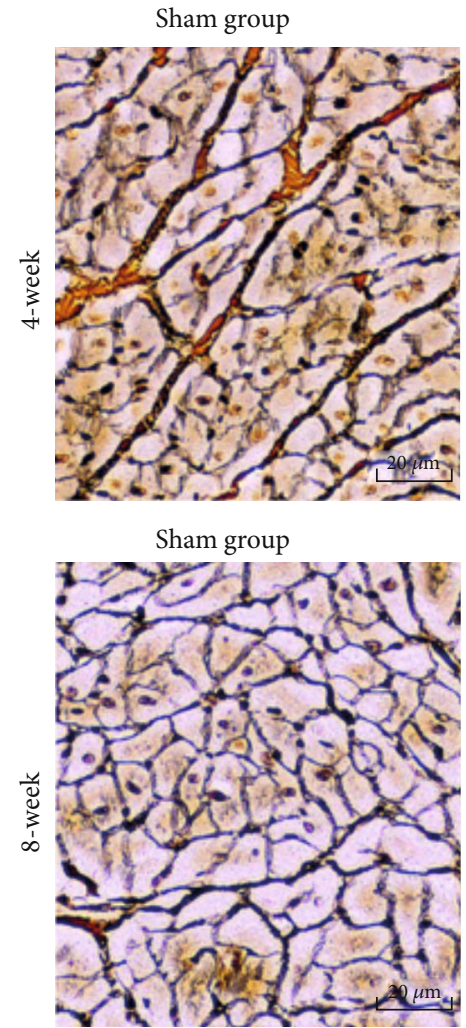
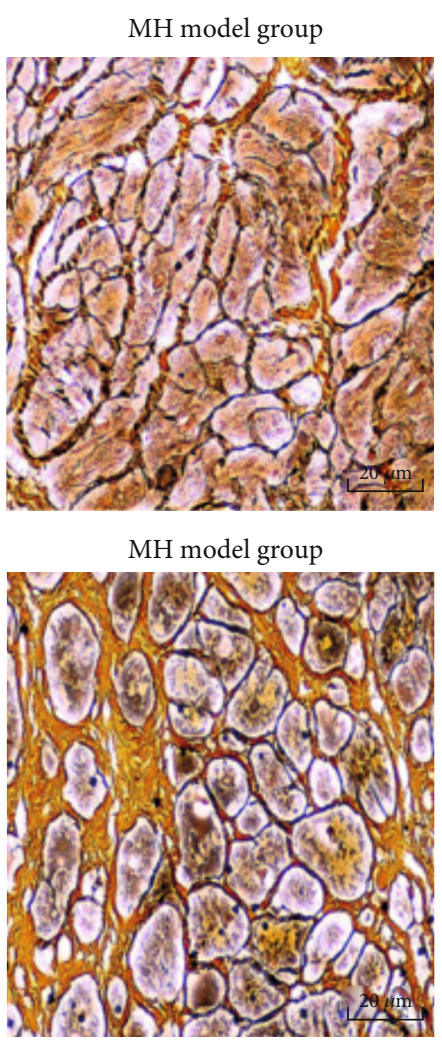

$\mathrm{MH}+$ Aspirin group

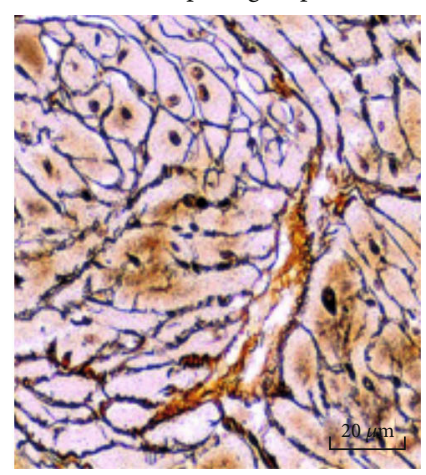

$\mathrm{MH}+$ Aspirin group

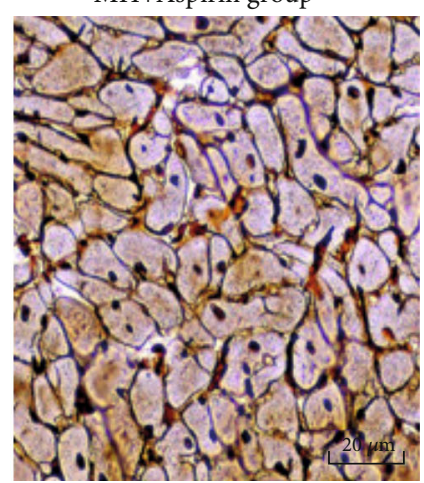

Figure 3: Pathological morphology of myocardial tissue in each group $(n=9)$. Myocardial tissues were collected from the sham group, MH model group, and $\mathrm{MH}+$ aspirin group at 4 weeks and 8 weeks after model establishment. Modified Gomori reticular fiber staining was performed to observe the fibers. Representative images were shown. Collagen fibers were yellow, reticular fibers were black, and cardiomyocytes were pale yellow or colorless. Magnification: 400x.

TABLE 5: Measurement of RVF-T (\%) in each group (mean \pm SD, $n=9)$.

\begin{tabular}{|c|c|c|}
\hline \multirow{2}{*}{ Groups } & \multicolumn{2}{|c|}{ RVF-T (\%) } \\
\hline & 4-week & 8-week \\
\hline Sham & $18.46 \pm 1.85$ & $19.30 \pm 1.75$ \\
\hline MH model & $12.99 \pm 3.72^{*}$ & $12.06 \pm 4.02^{*}$ \\
\hline $\mathrm{MH}+$ aspirin & $16.34 \pm 1.13^{\triangle}$ & $17.23 \pm 1.12^{\#}$ \\
\hline
\end{tabular}

Note: RVF-T: volume percentage of reticular fibers. ${ }^{*} P<0.01$ compared with the sham group at the same time point. ${ }^{\triangle} \mathrm{P}<0.05$ compared with the $\mathrm{MH}$ model group at the same time point. ${ }^{*} P<0.01$ compared with the $\mathrm{MH}$ model group at the same time point.

the release of many inflammatory factors, which can further stimulate collagen fibrosis and lead to myocardial fibrosis [22-26]. Meanwhile, they can activate thrombospondin-1 on platelet [27]. TGF- $\beta$ can promote the expression of extracellular matrix and inhibit the degradation of collagen fibers and participate in the occurrence of myocardial fibrosis [28]. Liang et al. [29] showed that specific COX-2 inhibitors significantly reduced the collagen fraction of $\mathrm{MH}$ rats and improved myocardial collagen remodeling and cardiac function. In this study, changes in myocardial pathology were observed by HE staining, Masson staining, and reticular fiber staining. At 4 and 8 weeks, the MH model groups showed disordered myocardial cell arrangement, hypertrophy, focal hyperplasia of collagen fibers, and marked reduction of reticular fibers. However, myocardial cells and fibers in the $\mathrm{MH}+$ aspirin groups did not change obviously. The results indicate that aspirin can significantly inhibit collagen fibrosis and cardiomyocyte hypertrophy, thereby inhibiting ventricular fibrosis. This is consistent with the study of Liang et al. [29].

Okumura et al. found that COX inhibitors lowered blood pressure in a kidney-clamp hypertension model [30]. Our study showed that SBP and DBP were significantly higher in the $\mathrm{MH}$ model groups whereas they were significantly reduced in the $\mathrm{MH}+$ aspirin groups, which is consistent with the results of Okumura et al. [30]. Previous studies [31, 32] have shown that the imbalance between $\mathrm{PGI}_{2}$ and $\mathrm{TXA}_{2}$ is involved in the occurrence and development of hypertension, which is the cause of the continuous development of blood pressure [33]. $\mathrm{PGI}_{2}$ is one of the main metabolites of arachidonic acid. It has the functions of relaxing blood vessels, lowering blood pressure, and increasing blood flow of organs [34]. PGI $_{2}$ can significantly reduce the pre- and postcirculation load and lower blood pressure, while TXA2 has the function of contracting blood vessels and raising blood pressure [35]. These two have antagonistic effects. Aspirin blocks the metabolism of arachidonic acid by inhibiting COX-1 [36] and significantly reduces the production of TXA2 without affecting the production of PGI2 [37], thereby lowering blood pressure. In this study, we found that at 8 weeks after model 

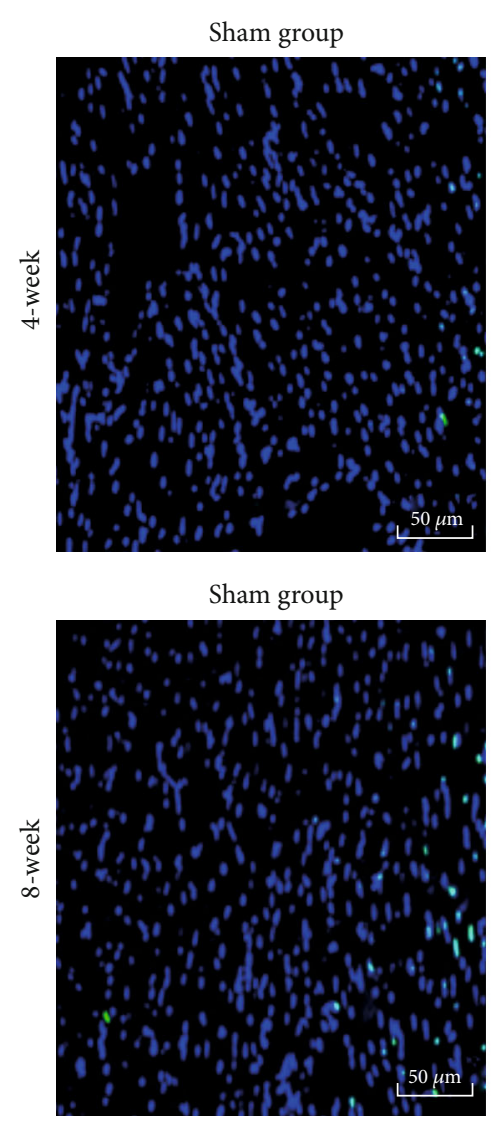
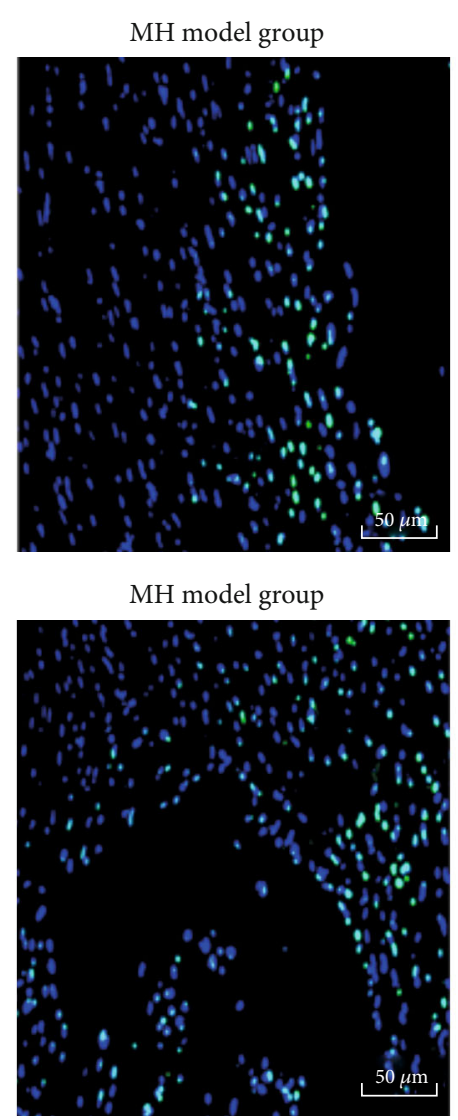
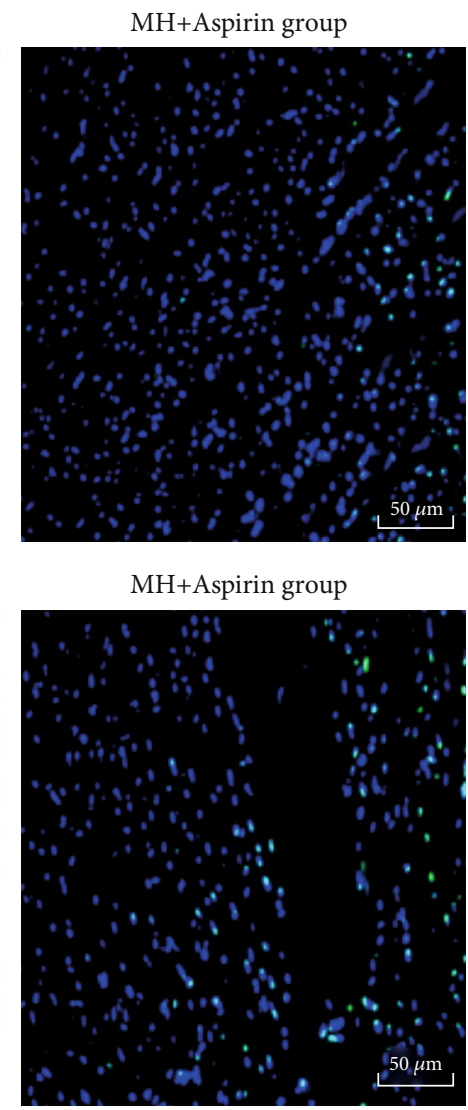

FIGURE 4: Cardiomyocyte apoptosis of rats in each group $(n=9)$. Myocardial tissues were collected from the sham group, MH model group, and $\mathrm{MH}+$ aspirin group at 4 weeks and 8 weeks after model establishment. Cardiomyocyte apoptosis was detected by TUNEL assay. Representative images were shown. The apoptosis is indicated as green fluorescence, and the nuclei are shown as blue fluorescence. Magnification: 400x.

TABLE 6: Comparison of myocardial cell apoptosis rates (\%) in each group (mean $\pm \mathrm{SD}, n=9$ ).

\begin{tabular}{lcc}
\hline Groups & 4-week (\%) & 8-week (\%) \\
\hline Sham & $5.06 \pm 0.54$ & $7.14 \pm 0.71$ \\
MH model & $12.52 \pm 0.77^{*}$ & $21.35 \pm 0.86^{*}$ \\
MH+aspirin & $8.93 \pm 0.56^{\triangle}$ & $11.64 \pm 0.79^{\triangle}$ \\
\hline
\end{tabular}

Note: ${ }^{*} P<0.05$ compared with the sham group at the same time point. ${ }^{\triangle} P<0.05$ compared with the MH model group at the same time point.

establishment, the $\mathrm{PGI}_{2}$ level in the $\mathrm{MH}$ model group was significantly decreased as compared with that in the sham group. The $\mathrm{PGI}_{2}$ level in the $\mathrm{MH}+$ aspirin group was significantly increased compared with that in the $\mathrm{MH}$ model group. This may be caused by the differences of different samples. Further studies are needed to clarify this. At 4 weeks and 8 weeks after model establishment, the TXA 2 level in the plasma and myocardial tissue was significantly increased the $\mathrm{MH}$ model groups while that was significantly decreased in the $\mathrm{MH}+$ aspirin group. This may partly explain the decrease of blood pressure in $\mathrm{MH}$ rats intervened with aspirin.

The imbalance between proinflammatory and antiinflammatory factors is one of important factors for the development of $\mathrm{MH}$ [38]. For example, the expression of inflammatory factors of TNF- $\alpha$ and IL-6 in the myocardial tissue of rats with $\mathrm{MH}$ was significantly increased [39]. Niu and Zhao [40] found that the expression of proinflammatory factors in the myocardial tissue was proportional to the level of collagen fibers in the myocardium, indicating that proinflammatory factors can promote the proliferation of collagen fibers in the myocardial tissue, leading to interstitial remodeling. Wang et al. [41] found that TNF- $\alpha$ induced $\mathrm{MH}$ in neonatal rats through the PI3K-IP3R-Ca ${ }^{2+}$ pathway. In this study, the TNF- $\alpha$ level was significantly increased in the $\mathrm{MH}$ model group whereas decreased in the $\mathrm{MH}+$ aspirin group. There was no significant change in IL-10 levels in the plasma and myocardial tissue. This result indicates that there may be imbalance of proinflammatory and antiinflammatory factors in $\mathrm{MH}$ rats and aspirin administration may reverse this imbalance.

Furthermore, we found that the myocardial cell apoptosis rate in the $\mathrm{MH}$ model group increased significantly at 4 and 8 weeks after model establishment, but the $\mathrm{MH}+$ aspirin group significantly decreased the apoptosis rate compared with the $\mathrm{MH}$ model group. According to the results, it can be speculated that myocardial apoptosis seriously affects the development of $\mathrm{MH}$. In the early stage of $\mathrm{MH}$, cardiomyocytes undergo compensatory cell hypertrophy under the stimulation of pressure overload. Due to the stimulation of 
TABLE 7: Changes of TNF- $\alpha$ in the serum and myocardial tissue in each group (mean \pm SD, $n=9(\mathrm{ng} / \mathrm{L})$ ).

\begin{tabular}{lccrr}
\hline Groups & \multicolumn{2}{c}{ Serum } & \multicolumn{2}{c}{ Myocardial tissue } \\
\hline Sham & $190.68 \pm 59.15$ & $210.86 \pm 46.38$ & 4-week & 8-week \\
MH model & $195.92 \pm 42.68$ & $212.49 \pm 65.91$ & $612.85 \pm 94.63$ & $658.82 \pm 192.55$ \\
MH+aspirin & $193.34 \pm 56.05$ & $236.07 \pm 70.96$ & $686.52 \pm 246.59^{*}$ & $972.89 \pm 265.93^{*}$ \\
\hline
\end{tabular}

Note: ${ }^{*} P<0.05$ compared with the sham group at the same time point. ${ }^{\triangle} P<0.05$ compared with the MH model group at the same time point.

TABLE 8: Changes of IL-10 in the serum and myocardial tissue in each group (mean $\pm \mathrm{SD}, n=9$ (ng/L)).

\begin{tabular}{|c|c|c|c|c|}
\hline \multirow{2}{*}{ Groups } & \multicolumn{2}{|c|}{ Serum } & \multicolumn{2}{|c|}{ Myocardial tissue } \\
\hline & 4-week & 8-week & 4-week & 8-week \\
\hline Sham & $58.91 \pm 12.31$ & $42.35 \pm 15.05$ & $245.75 \pm 67.12$ & $248.17 \pm 56.58$ \\
\hline MH model & $64.18 \pm 16.97$ & $43.13 \pm 13.72$ & $246.48 \pm 83.46$ & $256.71 \pm 48.97$ \\
\hline $\mathrm{MH}+$ aspirin & $61.78 \pm 12.16$ & $48.36 \pm 12.26$ & $250.13 \pm 59.46$ & $253.07 \pm 38.09$ \\
\hline
\end{tabular}

TABLE 9: Changes of $\mathrm{PGI}_{2}$ in the serum and myocardial tissue in each group (mean $\pm \mathrm{SD}, n=9$ (ng/L)).

\begin{tabular}{lcccr}
\hline Groups & & Serum & \multicolumn{2}{c}{ Myocardial tissue } \\
& 4-week & 8-week & 4-week & $172.71 \pm 9.14$ \\
Sham & $78.46 \pm 23.92$ & $86.18 \pm 21.63$ & $52.82 \pm 23.75$ & $147.69 \pm 9.27^{*}$ \\
MH model & $82.12 \pm 20.01$ & $69.54 \pm 22.02^{*}$ & $56.02 \pm 26.37$ & $179.53 \pm 7.29^{\triangle}$ \\
MH+aspirin & $75.19 \pm 24.48$ & $89.97 \pm 21.04^{\triangle}$ & $49.86 \pm 22.48$ & \\
\hline
\end{tabular}

Note: ${ }^{*} P<0.05$ compared with the sham group at the same time point. ${ }^{\triangle} P<0.05$ compared with the MH model group at the same time point.

TABLE 10: Changes of $\mathrm{TXA}_{2}$ in the serum and myocardial tissue in each group (mean $\pm \mathrm{SD}, n=9$ (ng/L)).

\begin{tabular}{lcccr}
\hline Groups & 4-week & Serum & \multicolumn{2}{c}{ Myocardial tissue } \\
& $328.59 \pm 105.09$ & $315.73 \pm 98.13$ & $1046.08 \pm 158.60$ & $962.74 \pm 130.12$ \\
Sham & $381.14 \pm 112.10^{*}$ & $434.31 \pm 91.47^{*}$ & $1168.21 \pm 263.42^{*}$ & $1250.49 \pm 247.55^{*}$ \\
MH model & $337.76 \pm 116.07^{\triangle}$ & $306.82 \pm 110.07^{\triangle}$ & $952.86 \pm 207.35^{\triangle}$ & $979.02 \pm 142.25^{\triangle}$ \\
MH+aspirin & & & 8-week
\end{tabular}

Note: ${ }^{*} P<0.05$ compared with the sham group at the same time point. ${ }^{\triangle} P<0.05$ compared with the MH model group at the same time point.

hypertrophic signals and in order to maintain myocardial structure and function, the myocardium initiates suicide mode and induces myocardial cell apoptosis; however, as time goes by, the stress overload stimulus persists, which may cause $\mathrm{MH}$ to decompensate, thereby increasing the myocardial cell apoptosis rate. In this process, aspirin can reduce the rate of myocardial apoptosis, delay the development of $\mathrm{MH}$, and improve ventricular function [42]. On the other hand, aspirin could reduce TNF- $\alpha$ and IL- 6 levels in patients with heart failure [43]. Studies have found that, in addition to the Caspase pathway, TNF- $\alpha$ can also induce cardiomyocyte apoptosis through the ceramide pathway $[44,45]$. Thus, TNF- $\alpha$ produced by the myocardial tissue may ultimately affect cardiac function by inducing myocardial apoptosis. In this study, TNF- $\alpha$ in the myocardial tissue of the $\mathrm{MH}$ model group was significantly increased at 4 weeks. Under chronic pressure overload, the rate of TNF- $\alpha$ induced cardiomyocyte apoptosis increased significantly at 8 weeks, confirming this statement. Moreover, the results of this study showed that aspirin significantly reduced the con- tent of TNF- $\alpha$ produced by the myocardial tissue, which is consistent with a previous study [43]. Therefore, it can be speculated that aspirin may reduce the rate of cardiomyocyte apoptosis by reducing the content of TNF- $\alpha$ in the myocardial tissue, thereby alleviating $\mathrm{MH}$ and improving ventricular function.

This study has some limitations. First, the sample size was relatively small. Second, the mechanism underlying the protective effects of aspirin on $\mathrm{MH}$ was not investigated. Third, the long-term effects of aspirin were not evaluated. Further studies are warranted.

\section{Conclusions}

In conclusion, our findings demonstrate that aspirin had anti-inflammatory effects, effectively reduced the expression of inflammatory factors, inhibited myocardial apoptosis, and had a certain protective effect on the prevention of $\mathrm{MH}$. This study may provide experimental evidence for the clinical treatment of $\mathrm{MH}$. 


\section{Data Availability}

The data that support the findings of this study are available on request from the corresponding author.

\section{Conflicts of Interest}

The authors declare that there is no conflict of interest regarding the publication of this paper.

\section{Authors' Contributions}

Xiaolong $\mathrm{Wu}$ and Minghui Wei contributed equally to this work. They were co-first authors of this article.

\section{Acknowledgments}

This study was supported by grants from the Inner Mongolia Natural Science Foundation (No. 2012MS1152).

\section{References}

[1] L. Li and L. Chen, "Study on the mechanism of myocardial hypertrophy," Hainan Medical Journal, vol. 19, no. 7, pp. 123-125, 2008.

[2] J. J. Hunter and K. R. Chien, "Signaling pathways for cardiac hypertrophy and failure," The New England Journal of Medicine, vol. 341, no. 17, pp. 1276-1283, 1999.

[3] W. Y. Chen, J. Hong, J. Gannon, R. Kakkar, and R. T. Lee, "Myocardial pressure overload induces systemic inflammation through endothelial cell IL-33," Proceedings of the National Academy of Sciences of the United States of America, vol. 112, no. 23, pp. 7249-7254, 2015.

[4] J. J. Mercadier, A. M. Lompre, B. Swynghedauw, and K. Schwartz, "Plasticity of myocardial phenotype during cardiac hypertrophy and failure," Bulletin de l'Académie Nationale de Médecine, vol. 177, no. 6, pp. 917-931, 1993.

[5] S. B. Haudek, G. E. Taffet, M. D. Schneider, and D. L. Mann, "TNF provokes cardiomyocyte apoptosis and cardiac remodeling through activation of multiple cell death pathways," The Journal of Clinical Investigation, vol. 117, no. 9, pp. 26922701, 2007.

[6] L. Gullestad, T. Ueland, L. E. Vinge, A. Finsen, A. Yndestad, and P. Aukrust, "Inflammatory cytokines in heart failure: mediators and markers," Cardiology, vol. 122, no. 1, pp. 23$35,2012$.

[7] J. Kuusisto, V. Kärjä, P. Sipola et al., "Low-grade inflammation and the phenotypic expression of myocardial fibrosis in hypertrophic cardiomyopathy," Heart, vol. 98, no. 13, pp. 10071013, 2012.

[8] M. Xue and L. Wu, "Effect of fosinopril on apoptosis of left ventricular myocardial cells in rats with pressure overloaded cardiac hypertrophy," Journal of Inner Mongolia Medical College, vol. 31, no. 3, pp. 204-208, 2009.

[9] J. R. Vane, Y. S. Bakhle, and R. M. Botting, "Cyclooxygenases 1 and 2," Annual Review of Pharmacology and Toxicology, vol. 38, no. 1, pp. 97-120, 1998.

[10] M. Crescente, L. Menke, M. V. Chan, P. C. Armstrong, and T. D. Warner, "Eicosanoids in platelets and the effect of their modulation by aspirin in the cardiovascular system (and beyond)," British Journal of Pharmacology, vol. 176, no. 8, pp. 988-999, 2019.
[11] P. Guillem-Llobat, M. Dovizio, A. Bruno et al., "Aspirin prevents colorectal cancer metastasis in mice by splitting the crosstalk between platelets and tumor cells," Oncotarget, vol. 7, no. 22, pp. 32462-32477, 2016.

[12] E. J. Mahdi, "Aspirin and its related non-steroidal antiinflammatory drugs," Libyan Journal of Medicine, vol. 8, no. 1 , article $21569,2013$.

[13] E. Miciak-Ławicka, B. Begier-Krasińska, A. Tykarski, and Z. Krasiński, "Does the timing of aspirin administration influence its antiplatelet effect - review of literature on chronotherapy," Polish Journal of Cardio-Thoracic Surgery, vol. 15, no. 2, pp. 125-129, 2018.

[14] A. Williams and C. H. Hennekens, "The role of aspirin in cardiovascular diseases-forgotten benefits?," Expert Opinion on Pharmacotherapy, vol. 5, no. 1, pp. 109-115, 2005.

[15] C. Zhang, F. Wang, Y. Zhang et al., "Celecoxib prevents pressure overload-induced cardiac hypertrophy and dysfunction by inhibiting inflammation, apoptosis and oxidative stress," Journal of Cellular and Molecular Medicine, vol. 20, no. 1, pp. 116-127, 2016.

[16] P. G. Anderson, M. F. Allard, G. D. Thomas, S. P. Bishop, and S. B. Digerness, "Increased ischemic injury but decreased hypoxic injury in hypertrophied rat hearts," Circulation Research, vol. 67, no. 4, pp. 948-959, 1990.

[17] E. Rosello-Lleti, M. Rivera, L. Martinez-Dolz et al., "Inflammatory activation and left ventricular mass in essential hypertension," American Journal of Hypertension, vol. 22, no. 4, pp. 444-450, 2009.

[18] F. Li and Y. Feng, Overview on Rat Cardiac Hypertrophy Model, China Modern Doctor, 2010.

[19] Y. Liang, G. Zhang, X. Tang, Y. Wang, Y. Liu, and J. Shi, "Systolic pressure and heart heavy index changes in whole process of overloaded heart hypertrophy," China Medical Herald, vol. 14, no. 14, pp. 16-19, 2017.

[20] X. Li, G. Wang, M. QiLi et al., "Aspirin reduces cardiac interstitial fibrosis by inhibiting Erk1/2-Serpine2 and P-Akt signalling pathways," Cellular Physiology \& Biochemistry, vol. 45, no. 5, pp. 1955-1965, 2018.

[21] J. Lindenfeld, A. D. Robertson, B. D. Lowes, and M. R. Bristow, "Aspirin impairs reverse myocardial remodeling in patients with heart failure treated with beta-blockers," Journal of the American College of Cardiology, vol. 38, no. 7, pp. 19501956, 2001.

[22] M. Chen and J. G. Geng, "P-selectin mediates adhesion of leukocytes, platelets, and cancer cells in inflammation, thrombosis, and cancer growth and metastasis," Archivum Immunologiae et Therapiae Experimentalis, vol. 54, no. 2, pp. 75-84, 2006.

[23] S. Danese and C. Fiocchi, "Platelet activation and the CD40/CD40 ligand pathway: mechanisms and implications for human disease," Critical Reviews in Immunology, vol. 25, no. 2, pp. 103-121, 2005.

[24] E. Lutgens, D. Lievens, L. Beckers, M. Donners, and M. Daemen, "CD40 and its ligand in atherosclerosis," Trends in Cardiovascular Medicine, vol. 17, no. 4, pp. 118-123, 2007.

[25] S. Lindemann, N. D. Tolley, D. A. Dixon et al., “Activated platelets mediate inflammatory signaling by regulated interleukin $1 \beta$ synthesis," Journal of Cell Biology, vol. 154, no. 3, pp. 485-490, 2001.

[26] K. S. S. Prasad, P. Andre, Y. Yan, and D. R. Phillips, “The platelet CD40L/GP IIb-IIIa axis in atherothrombotic disease," Current Opinion in Hematology, vol. 10, no. 5, pp. 356-361, 2003. 
[27] J. P. McMorrow, D. Crean, M. Gogarty et al., "Tumor necrosis factor inhibition modulates thrombospondin-1 expression in human inflammatory joint disease through altered NR4A2 activity," The American Journal of Pathology, vol. 183, no. 4, pp. 1243-1257, 2013.

[28] Y. Yue, K. Meng, Y. Pu, and X. Zhang, “Transforming growth factor beta (TGF- $\beta$ ) mediates cardiac fibrosis and induces diabetic cardiomyopathy," Diabetes Research \& Clinical Practice, vol. 133, pp. 124-130, 2017.

[29] Z. Liang, L. Zeng, Z. Ye, G. Chen, P. Wang, and M. Lu, “The influence of selective cyclooxygenase-2 inhibitor on left ventricular reconstitution in rats with pressure overloaded myocardial hypertrophy," Journal of Emergency Medicine, vol. 14, no. 6, pp. 479-481, 2005.

[30] T. Okumura, I. Hayashi, T. Ikezawa et al., "Cyclooxygenase-2 inhibitors attenuate increased blood pressure in renovascular hypertensive models, but not in deoxycorticosterone-salt hypertension," Hypertension Research, vol. 25, no. 6, pp. 927-938, 2002.

[31] E. D'Abril Ruiz-Leyja, R. Villalobos-Molina, J. J. López-Guerrero, I. A. Gallardo-Ortíz, S. E. Estrada-Soto, and M. Ibarra-Barajas, "Differential role of cyclooxygenase-1 and -2 on renal vasoconstriction to $\alpha_{1}$-adrenoceptor stimulation in normotensive and hypertensive rats," Life Sciences, vol. 93, no. 16, pp. 552-557, 2013.

[32] H. H. Zeng, L. J. Liu, Y. P. Huang, Y. P. Xie, and L. X. Wang, "Effect of celecoxib on pulmonary hypertension of chronic hypoxia and hypercapnic rats," Zhongguo Ying Yong Sheng Li Xue Za Zhi, vol. 27, no. 1, pp. 29-32, 2011.

[33] Y. Liu, X. Li, J. Cai, B. Zheng, and F. Xie, “A preliminary study of the anti-hypertensive mechanism of Xuefu Zhuyu decoction on SHR," Journal of Emergency in Traditional Chinese Medicine, vol. 24, no. 8, pp. 1376-1379, 2015.

[34] C. Benyahia, K. Boukais, I. Gomez et al., "A comparative study of $\mathrm{PGI}_{2}$ mimetics used clinically on the vasorelaxation of human pulmonary arteries and veins, role of the DPreceptor," Prostaglandins \& Other Lipid Mediators, vol. 107, no. 12, pp. 48-55, 2013.

[35] Z. Wang, C. Wang, W. Zhang, L. Wang, and T. Lei, "Changes of TXA2 and PGI2 during postoperative hypertensive crisis in patients with hypertensive intracerebral hemorrhage," Journal of Huazhong University of Science and Technology. Medical Sciences, vol. 28, no. 1, pp. 87-89, 2008.

[36] D. Stepensky and G. Rimon, "Competition between low-dose aspirin and other NSAIDs for COX-1 binding and its clinical consequences for the drugs' antiplatelet effects," Expert Opinion on Drug Metabolism \& Toxicology, vol. 11, no. 1, pp. 4152,2014

[37] I. M. Robbins, S. M. Kawut, D. Yung et al., "A study of aspirin and clopidogrel in idiopathic pulmonary arterial hypertension," The European Respiratory Journal, vol. 27, no. 3, pp. 578-584, 2006.

[38] L. Fang, A. H. Ellims, A. L. Beale, A. J. Taylor, A. Murphy, and A. M. Dart, "Systemic inflammation is associated with myocardial fibrosis, diastolic dysfunction, and cardiac hypertrophy in patients with hypertrophic cardiomyopathy," American Journal of Translational Research, vol. 9, no. 11, pp. 50635073, 2017.

[39] Y. Liang, G. Zhang, X. Tang, Y. Wang, Y. Liu, and G. He, “The significance of tumor necrosis factor- $\alpha$ and interleukin- 6 in experiment of myocardium hypertrophy," Journal of North Pharmacy, vol. 12, no. 6, pp. 97-98, 2015.
[40] X. Niu and L. Zhao, "The role of inflammatory factors and stress factors in the development of hypertensive left ventricular hypertrophy," Chinese Journal of Hypertension, vol. 6, no. 1, pp. 525-527, 2014.

[41] G. J. Wang, Y. S. Yao, and H. X. Wang, "Neonatal rat cardiomyocyte hypertrophy is induced by tumor necrosis factor- $\alpha$ through PI3K-IP_3R-Ca (2+) pathways," Chinese Journal of Pathophysiology, vol. 32, pp. 21-26, 2016.

[42] R. Wu, D. Yin, N. Sadekova, C. F. Deschepper, J. de Champlain, and H. Girouard, "Protective effects of aspirin from cardiac hypertrophy and oxidative stress in cardiomyopathic hamsters," Oxidative Medicine and Cellular Longevity, vol. 2012, Article ID 761710, 8 pages, 2012.

[43] C. Liu and B. Bai, "Effect of aspirin on IL6 and TNF in patients with heart failure," China Pharmaceuticals, vol. 11, pp. 43-44, 2012.

[44] L. Xu, S. Gao, and R. Zhao, "Research progress of Livin and caspase-3 and malignant tumors," Clinical Metadata, vol. 27, no. 11, pp. 997-1001, 2012.

[45] M. Zhao, Y. Qin, L. Lu et al., "Preliminary study of necroptosis in cardiac hypertrophy induced by pressure overload," Sheng wu yi xue gong cheng xue za zhi = Journal of biomedical engineering $=$ Shengwu yixue gongchengxue zazhi, vol. 32, no. 3, pp. 618-623, 2015. 